Series A

\author{
I. MATHEMATICA
}

424

\title{
DUALITY FOR COHERENT SHEAVES ON ANALYTIC MANIFOLDS
}

BY

KALEVI SUOMINEN

HELSINKI 1968

S U O M A L A I EN TIEDEAKATEM I A

doi:10.5186/aasfm.1969.424 
Communicated 4 April 1968 by K. T. Virtanen and Lauri Ilyrberg 


\section{Introduction}

The duality for the cohomology of sheaves arose first in connection with the Riemann-Roch theorem. The proof of the classical Riemann-Roch formula for a compact non-singular analytic curve $X$ depends on the fact that the spaces

$$
H^{1}(X ; \mathscr{L}) \text { and } H^{0}\left(X ; \mathscr{L}^{-1} \otimes \omega\right)
$$

are dual for all invertible sheaves $\mathscr{L}$ on $X$. Here $\omega$ denotes the sheaf of germs of holomorphic differential forms on $X$. This duality theorem was generalized by Serre for locally free sheaves of finite type on compact analytic manifolds (Serre [5]). His proof depends on the use of distributions for the construction of resolutions of sheaves.

Later, Grothendieck generalized the duality theorem in several ways (cf. Hartshorne [3] for the algebraic case). An essential point of his method is the notion of residual complex which replaces distributions. On the other hand, Serre observed that his proof may be extended to coherent analytic sheaves which are not locally free, provided it can be shown that the ring of germs of $C^{\infty}$-functions at a point of $C^{n}$ is flat over the ring of germs of holomorphic functions. Malgrange has proved this by means of division of distributions, thus extending Serre's duality theorem (Malgrange [4]).

In this paper, we prove the duality theorem for coherent analytic sheaves, without division of distributions following a suggestion of Grothendieck according to an oral communication by Malgrange. The proof depends on localisation and on spectral sequences for passing to the global case. In addition, we calculate the cohomology groups with compact supports locally by an inductive argument. Hence distributions are wholly dispensed with.

\section{Ext with supports}

Let $X$ by a topological space and $\mathscr{\theta}_{X}$ a sheaf of rings on $X$. Let $\mathcal{F}$ and $C_{\mathcal{G}}$ be $\mathscr{\Theta}_{X}$-Modules.

Let $\varphi$ be a family of supports on $X(G, p .133)$. We denote by $\mathrm{Hom}_{\varphi, O_{X}}$ $\left(\mathcal{F}, C_{9}\right)$ the subgroup of $\operatorname{Hom}_{O_{X}}(\mathcal{F}, \mathscr{G})$ which consists of homomorphisms 
with support in $\varphi$. In the abelian category of $\mathscr{C}_{\boldsymbol{X}}$-Modules, each object has a injective resolution $(G$, p. 261). Hence the derived functors of

$$
C_{\mathcal{H}} \mapsto \operatorname{Hom}_{\varphi, O_{X}}\left(\mathcal{F}, C_{\mathcal{G}}\right)
$$

exist. In fact, let $\varphi^{\cdot}$ be a resolution of $\varphi_{1}$ by injective $\mathscr{C}_{X}$-Modules. Then the cohomology groups $H^{n}\left(\operatorname{Hom}_{4}, O_{X}\left(\mathcal{F}, \mathcal{Y}^{\circ}\right)\right)$ are independent of the resolution 9 . They depend functorially on $\mathcal{F}$ and $C_{1}$, and are denoted by $\operatorname{Ext}_{\varphi, O_{X}}^{n}\left(\mathcal{F}, \mathscr{G}_{)}\right)$. Clearly $\operatorname{Ext}_{\varphi, O_{X}}^{0}\left(\mathcal{F}, \varphi_{\mathcal{G}}\right)=\operatorname{Hom}_{\varphi, O_{X}}\left(\mathcal{F}, \mathcal{G}_{1}\right)$, since the latter is left exact.

In the case $\mathcal{F}=\Theta_{X}$ we have

$$
\operatorname{Hom}_{r, O_{X}}\left(\Theta_{X}, \varrho_{l}\right)=\Gamma_{\varphi}\left(C_{l}\right)
$$

and hence

$$
\operatorname{Ext}_{\varphi, O_{X}}^{n}\left(\mathscr{C}_{X}, \varphi_{Y}\right)=H_{\varphi}^{n}\left(X ; \varphi_{l}\right)
$$

In what follows we are mainly concerned with two families of supports. First, if $\varphi$ consists of all closed subsets of $X$, we obtain the ordinary Ext groups

$$
\operatorname{Ext}_{O X}^{n}\left(\mathcal{F}, \varphi_{1}\right)
$$

(cf. $G$, p. 263). In this case the symbol $\varphi$ is traditionally omitted.

In the second place, we shall consider the family $c$ of all closed compact subsets of $X$. If $X$ is a locally compact Hausdorff space, then the family $c$ is paracompactifying $(G, \mathrm{p} .150)$.

Let $U$ be an open subset of $X$. Then the restriction map

$$
\operatorname{Hom}_{O_{X}}\left(\mathcal{F}, C_{J}\right) \rightarrow \operatorname{Hom}_{O_{X \mid U}}(\mathcal{F} \mid U, C, U)
$$

induces homomorphisms of derived functors

$$
\operatorname{Ext}_{O_{X}}^{n}\left(\mathcal{F}, \varrho_{Y}\right) \rightarrow \operatorname{Ext}_{O_{X} \mid U}^{n}\left(\mathcal{F}\left|U, \varrho_{I}\right| U\right)
$$

Thus

$$
U \rightarrow \operatorname{Ext}_{O_{X} \mid U}^{n}(\mathcal{F}|U, \mathcal{C}| U)
$$

becomes a presheaf which is denoted by $\operatorname{ext}_{O_{X}}^{n}\left(\mathcal{F}, C_{1}\right)$.

Let $\mathfrak{U}$ be an open cover of $X$. For each presheaf $\mathscr{L}$ of abelian groups on $X$, we denote by $C^{*}(\mathfrak{H} ; \mathscr{L})$ the alternating cochain complex of the nerve of $\mathfrak{U}$ with coefficients defined by $\mathscr{L}$. This complex may be augmented by a canonical homomorphism $\mathscr{L}(X) \rightarrow C^{0}(\mathfrak{U} ; \mathscr{L})$. The cohomology groups of $C^{*}(\mathfrak{u} ; \mathscr{L})$ are denoted by $H^{*}(\mathfrak{U} ; \mathscr{L})$. 
Proposition 1.1. Let $\exists$ and $C_{\mathcal{J}}$ be ${ }^{\mathscr{C}_{X}}$-Modules. Then, for each open cover $\mathfrak{U}$ of $X$, there is a spectral sequence $E$ with

$$
E_{1}^{p q}=C^{p}\left(\mathfrak{l} ; \operatorname{ext}_{\partial_{X}}^{q}\left(\mathcal{F}, \varrho_{\jmath}\right)\right)
$$

converging to $\operatorname{Ext}_{\mathrm{O}_{X}}\left(\overline{7}, C_{9}\right)$.

Proof: Let $\varphi^{\circ}$ be an injective resolution of $\varphi_{l}$, and denote by $\mathcal{L}^{*}$ the complex of sheaves $\operatorname{M}_{0 m_{X}}\left(\mathcal{F}, 9^{\circ}\right)$. Let us consider the double complex $C^{\cdot}$ with

$$
C^{p q}=C^{p}\left(\mathfrak{H} ; \mathscr{L}^{q}\right)
$$

(cf. $G$, p. 210). The first spectral sequence of $C^{\cdot *}$ converges to $H^{*}\left(C^{*}\left(\mathfrak{H} ; \mathcal{C}^{\bullet}\right)\right)$, and

$$
{ }^{\prime} E_{1}^{p q}=C^{p}\left(\mathfrak{U} ; H^{q}\left(\mathscr{C}^{\bullet}\right)\right)
$$

where $H^{q}\left(\mathscr{L}^{\bullet}\right)$ denotes the presheaf $U \rightarrow H^{q}(\Gamma(U ; \mathscr{C} \cdot))$. Clearly $H^{q}(\mathscr{L} \cdot)=$ $\operatorname{ext}_{O_{X}}\left(\mathcal{F}, \varphi_{j}\right)$.

On the other hand, the sheaves $\mathscr{L}^{q}=\operatorname{Com}_{\mathrm{O}_{X}}\left({ }^{7}, 9^{q}\right)$ are flabby $(G$, p. 264). Then the second spectral sequence of $C^{*}$ degenerates, and the augmentation

$$
\Gamma\left(\mathscr{L}^{\bullet}\right) \rightarrow C^{\cdot}\left(\mathfrak{H} ; \mathscr{L}^{\bullet}\right)
$$

induces an isomorphism on the cohomology of simple complexes

$$
\operatorname{Ext}_{O_{X}}^{n}\left(\mathcal{F}, C_{Y}\right) \simeq H^{n}\left(C^{*}\left(\mathfrak{H} ; \mathscr{L}^{\cdot}\right)\right)
$$

Hence the first spectral sequence of $C^{\cdot}$ has the desired properties.

In the rest of this section, we shall assume that $X$ is a locally compact Hausdorff space. Hence the family $c$ is paracompactifying. If $U$ is an open subset of $X$, we continue to denote by $c$ the family $c \mid U$ of compact sets contained in $U$.

Let $\mathscr{L}$ be a sheaf of abelian groups on $X$. For each open subset $U$ of $X$, we set $\mathscr{L}_{c}(U)=\Gamma_{c}(U ; \mathscr{L})$. Each section $s \in \Gamma_{c}(U ; \mathcal{L})$ may be continuously extended to $X$ by zero. Hence there are natural injections $\mathscr{L}_{c}(U) \rightarrow \mathscr{L}_{c}(V)$ for open sets $V \supset U$. Thus $\mathscr{L}_{c}$ may be considered as a precosheaf.

Let $\mathfrak{U}$ be an open cover of $X$. For each precosheaf $\mathfrak{A}$ of abelian groups on $X$, we denote by $C .(11 ; \mathfrak{A})$ the alternating chain complex of the nerve of $\mathfrak{U}$ with coefficients defined by $\mathfrak{A}$. This complex may be augmented by a canonical homomorphism $C_{\mathbf{0}}(\mathfrak{H} ; \mathfrak{A}) \rightarrow \mathfrak{A}(X)$. The homology groups of $C .(\mathfrak{U} ; \mathfrak{A})$ are denoted by $H .(\mathfrak{U} ; \mathfrak{U})$. 
Let $\mathcal{L}^{\cdot}$ be a complex of sheaves on $X$. Let us consider the double complex $C^{\cdot}$ with

$$
C^{p q}=C_{-p}\left(\mathfrak{l} ; \mathscr{L}_{\mathrm{c}}^{q}\right) .
$$

The first filtration of $C^{\cdot \cdot}$ is regular, giving rise to a convergent spectral sequence with

$$
{ }^{\prime} E_{1}^{p q}=C_{-p}\left(\mathfrak{U} ; H^{q}\left(\mathscr{L}_{c}^{*}\right)\right) .
$$

Here $H^{q}\left(\mathscr{L}_{c}^{\cdot}\right)$ denotes the precosheaf $U \mapsto H^{q}\left(\Gamma_{c}\left(U ; \mathscr{L}^{\bullet}\right)\right)$.

The convergence of the second spectral sequence is not obvious. We shall only consider the case in which the sheaves $\mathscr{L}^{q}$ are $c$-soft.

Lemma 1.2. Let $\mathfrak{U}=\left(U_{i}\right)_{0 \leq i \leq n}$ be a finite open cover of $X$, and let $\mathscr{L}$ be a $c$-soft sheaf on $X$. Then the complex

$$
\text { C. }\left(\mathfrak{U} ; \mathscr{L}_{c}\right)
$$

is acyclic in dimensions $p>0$, and the augmentation map induces an isomorphism

$$
H_{0}\left(\mathfrak{H} ; \mathscr{L}_{c}\right) \rightarrow \Gamma_{c}(\mathscr{L}) .
$$

Proof: Let $S_{p}$ be the family of $(p+1)$-tuples $s=\left(i_{0}, \ldots, i_{p}\right)$ such that $0 \leq i_{0}<i_{1}<\ldots<i_{p} \leq n$. For each $s \in S_{p}$, we set

$$
U_{s}=U_{i_{0} \cdots i_{p}}=U_{i_{0}} \cap \ldots \cap U_{i_{p}},
$$

and denote by $\mathscr{L}_{s}=\mathscr{L}_{i_{0} \ldots i_{p}}$ the sheaf $\mathscr{L}_{U_{s}}$, extension of $\mathscr{L} \mid U_{s}$ by zero $\left(G\right.$, p. 140). Then the sheaves $\mathscr{L}_{s}$, as well as their direct sums

$$
\mathscr{C}_{p}\left(\mathfrak{H} ; \mathscr{L}^{\prime}\right)=\sum_{s \in S_{p}} \mathscr{L}_{s},
$$

are $c$-soft $(G$, II. 3.5). For each $p>0$, we define the differential

$$
d: \mathscr{C}_{p}(\mathfrak{H} ; \mathscr{L}) \rightarrow \mathcal{C}_{p-1}(\mathfrak{H} ; \mathscr{L})
$$

by $d \mid \mathscr{K}_{s}=\sum_{k=0}^{p}(-1)^{k} j_{k} \quad$ where

$$
j_{k}: \mathscr{L}_{i_{0} \cdots i_{p}} \rightarrow \mathscr{L}_{i_{0} \cdots \hat{i}_{k} \cdots i_{p}}
$$

is the inclusion map. In addition, the inclusions $\mathscr{L}_{i_{0}} \rightarrow \mathscr{L}$ define an aug. mentation map $\mathscr{C}_{\mathbf{0}}(\mathfrak{u} ; \mathscr{L}) \rightarrow \mathscr{L}$.

We claim that the complex $\mathcal{C} .(\mathfrak{U} ; \mathscr{L})$ is a resolution of $\mathscr{L}$. Let $x$ be a point of $X$, and let $s=\left(i_{0}, \ldots, i_{p}\right)$ be the sequence of integers $i$ such that $x \in U_{i}$. Then $\mathscr{C} .\left(\mathfrak{U} ; \mathscr{L}_{\mathfrak{x}}\right)_{\boldsymbol{x}}$ is the alternating chain complex of the 
$p$-simplex $s$ with coefficients $\mathscr{L}_{x}$. This proves the exactness of the augmented complex of $c$-soft sheaves

$$
0 \rightarrow \mathscr{C}_{n}(\mathfrak{u} ; \mathscr{L}) \stackrel{d}{\rightarrow} \ldots \stackrel{d}{\rightarrow} \mathscr{C}_{0}(\mathfrak{H} ; \mathscr{L}) \rightarrow \mathscr{L} \rightarrow 0
$$

Applying the functor $\Gamma_{c}$, we obtain the exact sequence

$$
0 \rightarrow C_{n}\left(\mathfrak{H} ; \mathscr{L}_{c}\right) \rightarrow \ldots \rightarrow C_{0}\left(\mathfrak{U} ; \mathscr{L}_{c}\right) \rightarrow \Gamma_{c}(\mathscr{L}) \rightarrow 0
$$

(G, p. 154). This concludes the proof.

The discussion of the double complex $C .\left(\mathfrak{U} ; \mathscr{L}_{c}\right)$ may now be completed:

Theorem 1.3. Let $\mathfrak{U}$ be an open cover of a locally compact Hausdorff space $X$. Let $\mathscr{L}^{\cdot}$ be a complex of c-soft sheaves on $X$. Then there is a spectral sequence ${ }_{c} E$ with

$$
{ }_{c} E_{1}^{p q}=C_{-p}\left(\mathfrak{U} ; H^{q}\left(\mathscr{L}_{\mathbf{c}}\right)\right)
$$

converging to $H^{*}\left(\Gamma_{c}\left(X ; \mathscr{L}^{\bullet}\right)\right)$.

Proof: Let $\mathfrak{B}$ be a finite subfamily of $\mathfrak{U}$. Then the second spectral sequence of the double complex

$$
C_{\mathfrak{B}}^{\cdot \cdot}=C \cdot\left(\mathfrak{B} ; \mathscr{L}_{c}^{\cdot}\right)
$$

degenerates by Lemma 1.2. Hence the augmentation map

$$
C_{\mathfrak{B}}^{\cdot} \rightarrow \Gamma_{c}(V ; \mathscr{L} \cdot)
$$

where $V=U \mathfrak{F}$, induces an isomorphism on cohomology.

The complex $C^{\cdot *}$ is the direct limit of the subcomplexes $C_{\mathfrak{*}}^{\cdot *}$, and also

$$
\Gamma_{c}\left(X ; \mathscr{L}^{\bullet}\right)=\lim _{\rightarrow} \Gamma_{c}\left(V ; \mathscr{L}^{*}\right)
$$

By the exactness of the functor $\underset{\rightarrow}{\lim }$, we infer that the augmentation map

$$
C^{\cdot \bullet} \rightarrow \Gamma_{c}(X ; \mathscr{L} \cdot)
$$

induces an isomorphism on cohomology

$$
H^{*}\left(C^{*}\right) \stackrel{\sim}{\rightarrow} H^{*}\left(\Gamma_{c}\left(X ; \mathscr{L}^{*}\right)\right)
$$

Hence the first spectral sequence of $C^{\cdot \cdot}$ has the desired properties.

Now we return to the situation at the beginning of this section. Let $U$ be an open subset of $X$. There is a canonical injection

$$
\operatorname{Hom}_{c, O_{X} \mid U}\left(\mathcal{F}\left|U, \mathscr{C}_{\mathcal{J}}\right| U\right) \rightarrow \operatorname{Hom}_{c, O_{X}}\left(\mathcal{F}, \mathscr{C}_{\mathcal{J}}\right)
$$


which induces homomorphisms of derived functors

$$
\operatorname{Ext}_{c, O_{X} \mid U}^{n}\left(\mathcal{F}\left|U, C_{Y}\right| U\right) \rightarrow \operatorname{Ext}_{c, O_{X}}^{n}\left(\mathcal{F}, C_{l}\right) .
$$

Hence

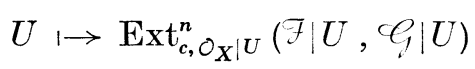

becomes a precosheaf which is denoted by $\operatorname{ext}_{c, O_{X}}^{n}\left(\mathcal{F}, C_{J}\right)$. Clearly, this precosheaf is $\left.H^{n}\left(\operatorname{Com}_{X}(\mathcal{F},)^{\circ}\right)_{c}\right)$ for any injective resolution $y^{\circ}$ of $C_{1}$. Since the sheaves $\mathcal{M}_{0 m_{X}}(\mathcal{F}, Y q)$ are are flabby, and hence $c$-soft, we may apply Theorem 1.3 to the complex $\operatorname{Com}_{\mathrm{X}}\left(7,9^{\circ}\right)$ obtaining

Corollary 1.4. Let $\mathcal{F}$ and $C_{\mathcal{J}}$ be $\mathscr{Q}_{X^{-}}$Modules. Then, for each open cover $\mathfrak{H}$ of $X$, there is a spectral sequence ${ }_{c} E$ with

$$
{ }_{c} E_{1}^{p q}=C_{-p}\left(\mathfrak{H} ; \operatorname{ext}_{c, O_{X}}^{q}\left(\mathcal{F}, \varrho_{\urcorner}\right)\right)
$$

converging to $\operatorname{Ext}_{c, O_{X}}\left(\mp, \varphi_{1}\right)$.

\section{Composition product for Ext}

Let $\mathcal{F}, \mathscr{C}_{\mathcal{J}}$, and $\mathcal{X}$ be $\mathscr{\Theta}_{X}$-Modules. For any families of supports $\varphi$ and $\psi$, the composition of homomorphisms defines a linear map

$$
\operatorname{Hom}_{\varphi, O_{X}}\left(\mathcal{F}, C_{Y}\right) \otimes \operatorname{Hom}_{\psi}, O_{X}\left(C_{,}, X\right) \rightarrow \operatorname{Hom}_{\varphi \cap \psi, O_{X}}(\mathcal{F}, \mathcal{X}) \text {. }
$$

We propose to show how this map induces a canonical composition product for the groups Ext with supports (cf. Cartier [2]).

Let $\%^{\circ}$ and $\%^{\circ}$ be injective resolutions of $\mathcal{F}$ and $C_{\mathcal{F}}$ in the category of ${ }^{(}$-Modules. We define a complex $\operatorname{Hom}_{q, O_{X}}\left(9^{\circ}, 9^{\circ}\right)$ by

$$
\operatorname{Hom}_{\varphi, O_{X}}^{q}\left(9^{\bullet}\right)=\prod_{p=0}^{\infty} \operatorname{Hom}_{\varphi, O_{X}}\left(9 p, \zeta^{\natural} q+p\right) .
$$

The differential is given by

$$
d f=\left(d^{p+q} \circ f^{p}+(-1)^{q+1} f^{p+1} \circ d^{p}\right)_{p \geq 0}
$$

for $\left.f=\left(f^{p}\right) \in \operatorname{Hom}_{q, O_{X}}^{q}\left(\mathscr{G}^{*},\right)^{*}\right)$. The $q$-cycles of this complex are chain maps of degree $q$ from $\%$ to $\%$, and $q$-boundaries are those which are homotopic to zero.

We shall show that the complex $\operatorname{Hom}_{\varphi, O_{X}}^{\cdot}\left(\%^{\circ}, ?^{*}\right)$ is cohomologically equivalent to $\operatorname{Hom}_{\varphi, O_{X}}\left(7,9^{\circ}\right)$.

We first prove 
Lemma 2.1. Let 97 be a sub-Module of an $\mathscr{Q}_{X^{-M}}$ Module 7 . Let 9 be an injective $\mathscr{Q}_{X^{-}}$Module. Then each $\mathscr{Q}_{X^{-} \text {Module homomorphism } f: ~} \rightarrow \rightarrow 9$ may be extended to a homomorphism $g: ~ \nearrow \rightarrow 9$ such that supp $g=\operatorname{supp} f$.

Proof: Let $U$ be the complement of $F=\operatorname{supp} f$ in $X$. Denote by $m_{U}$ the sheaf which induces $m \mid U$ on $U$ and the zero sheaf on $F(G, p$. 138). It is clear that $m_{U}$ is a sub- $\mathscr{C}_{X}$-Module of $m$ and $f\left(m_{U}\right)=0$. Since $9 l_{U} \cap 9 m=9 m_{U}$, it is possible to extend $f$ to a homomorphism $f^{\prime}: \nearrow_{U} \cup 9 m \rightarrow 9$ by defining $f^{\prime}=0$ in $\eta_{U}$. Then, for each extension $g: 9 l \rightarrow 9$ of $f^{\prime}$ it is true that $\operatorname{supp} g=F$. This completes the proof.

Returning to our previous notation, we now easily see by means of Lemma 2.1 (cf. Cartier [2], Théoreme 1) that the canonical homomorphism

$$
\operatorname{Hom}_{\varphi, O_{X}}\left(g^{\cdot}, g^{*}\right) \rightarrow \operatorname{Hom}_{\varphi, O_{X}}\left(\mathcal{F}, 9^{*}\right)
$$

induces an isomorphism on cohomology

$$
H^{q}\left(\operatorname{Hom}_{\varphi, O_{X}}^{\cdot}\left(\mathscr{G}^{\cdot}, g^{\cdot}\right)\right) \stackrel{\sim}{\rightarrow} \operatorname{Ext}_{\varphi, O_{X}}^{q}\left(\mathcal{F}, \varphi^{g}\right)
$$

Now let $\mathcal{K}$ be an injective resolution of the third $\mathscr{O}_{X}$-Module $\mathscr{C}$. Then the composition defines a map

$$
\operatorname{Hom}_{\varphi, O_{X}}^{\cdot}\left(9^{\cdot}, 9^{*}\right) \otimes \operatorname{Hom}_{\psi, O_{X}}^{\cdot}\left(9^{\cdot}, \mathcal{K}^{*}\right) \rightarrow \operatorname{Hom}_{\varphi \cap \psi, O_{X}}^{\cdot}\left(g^{\cdot}, \mathcal{K}^{*}\right)
$$

which is compatible with the differentials. From what has preceded it will be seen that this induces products

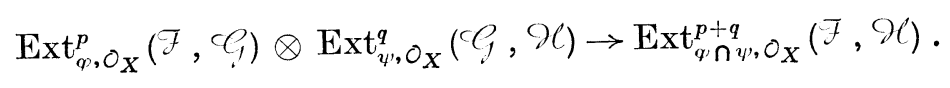

It is clear that this composition product for the groups Ext with supports is associative.

In the rest of this section we shall consider the case in which $\varphi$ is the family of all closed subsets of $X$ and $\psi=c$. We also assume that $X$ is a locally compact Hausdorff space. Then we have the composition products

$$
\operatorname{Ext}_{\mathcal{O}_{X}}^{p}\left(\mathcal{F}, \mathscr{G}_{\mathcal{J}} \otimes \operatorname{Ext}_{c, O_{X}}^{a}(\mathscr{C}, \mathcal{X}) \rightarrow \operatorname{Ext}_{c, O_{X}}^{p+q}(\mathcal{F}, \mathcal{X})\right.
$$

which clearly define a pairing

$$
\operatorname{ext}_{\mathcal{O}_{X}}^{p}\left(\mathcal{F}, \mathscr{C}_{\mathcal{J}}\right) \otimes \operatorname{ext}_{c, O_{X}}^{q}\left(\mathscr{C}_{\mathcal{J}}, \mathcal{X}\right) \rightarrow \operatorname{ext}_{c, O_{X}}^{p+q}(\mathcal{F}, \mathcal{X})
$$

from a presheaf and a precosheaf to a precosheaf in an obvious sense.

Recalling the spectral sequences of Proposition 1.1 and Corollary 1.4, we may now state 
Theorem 2.2. Let $\mathcal{F}, \mathscr{G}$, and $\mathscr{C}$ be $\mathscr{C}_{X}$-Modules. Then, for each open cover $\mathfrak{U}$ of $X$, there is a pairing of spectral sequences $E \otimes{ }_{c} E \rightarrow_{c} E$ compatible with the composition product of abutments

$$
\operatorname{Ext}_{O_{X}}(\mathcal{F}, \mathscr{G}) \otimes \operatorname{Ext}_{c, O_{X}}(\mathcal{G}, \mathcal{X}) \rightarrow \operatorname{Ext}_{c, O_{X}}(\mathcal{F}, \mathcal{X})
$$

such that the pairing of $E_{1}$-terms

$C^{p_{1}}\left(\mathfrak{H} ; \operatorname{ext}_{O_{X}}^{q_{1}}\left(\mathcal{J}, \varrho_{1}\right)\right) \otimes \mathrm{C}_{-p_{\mathfrak{z}}}\left(\mathfrak{H} ; \operatorname{ext}_{c, O_{X}}^{q_{2}}\left(C_{\mathcal{G}}, \mathcal{C}\right)\right) \rightarrow C_{-p_{1}-p_{2}}\left(\mathfrak{H} ; \operatorname{ext}_{c, O_{X}}^{q_{1}+q_{2}}(\mathcal{J}, \mathcal{C})\right)$

is a cap product on cochains and chains (e.g. Spanier [6], p. 254).

Proof: Let $9^{\circ}, 9^{\circ}$, and $\mathcal{K}$ be injective resolutions of $\mathcal{F}, \mathcal{C}$, and ' $\mathcal{C}$, respectively. Let $x_{0 m_{X}}\left(g^{\circ}, g^{\circ}\right)$ denote the complex of sheaves defi-

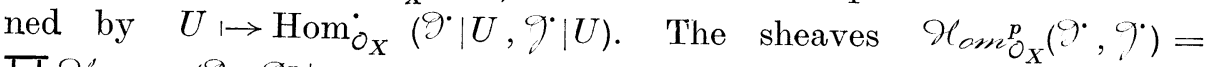
$\prod \operatorname{Com}_{\mathrm{o}_{X}}\left(9^{q}, g^{p+q}\right)$ are flabby. Hence the double complex $C_{1}=$ $C^{*}\left(\mathfrak{H} ; \mathcal{M}^{\circ \dot{c}_{C_{X}}}\left(9^{*}, 9^{\circ}\right)\right)$ may be used for the construction of the spectral sequence of $\operatorname{Ext}_{0 X}\left(\mathcal{F}, \varphi_{\mathcal{G}}\right)$.

For the same reason, the spectral sequences of $\operatorname{Ext}_{c, O_{X}}\left(C_{Y}, C^{\prime}\right)$ and $\operatorname{Ext}_{c, O_{X}}(\mathcal{F}, \mathcal{C})$ may be defined by means of the double complexes $C_{2}=$

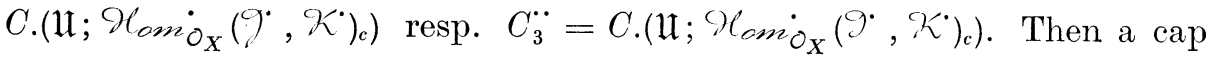
product on cochains and chains induces a pairing of double complexes

$$
C_{1}^{\cdot \bullet} \otimes C_{2}^{\cdot \cdot} \rightarrow C_{3}^{\cdot \cdot}
$$

Since a cap product is compatible with augmentations, this pairing defines a pairing of spectral sequences which is compatible with the composition product of abutments. This completes the proof.

\section{Cohomology of the sheaves $\Omega_{X}^{p}$}

From now on we shall assume that $X$ is a complex analytic manifold of complex dimension $n$, and denote by $\mathscr{C}_{X}$ the sheaf of germs of holomorphic functions on $X$. Since $X$ is a locally compact Hausdorff space, all the results of sections 1 and 2 may be applied here.

Let $\Omega_{X}^{p}$ denote the sheaf of germs of holomorphic $p$-differential forms on $X$, in particular $\mathscr{O}_{X}=\Omega_{X}^{0}$. There exist canonical resolutions of the following type

$$
0 \rightarrow \Omega_{X}^{p} \rightarrow \mathscr{C E}_{X}^{p 0} \stackrel{d^{\prime \prime}}{\rightarrow} \mathscr{C}_{X}^{p 1} \stackrel{d^{\prime \prime}}{\rightarrow} \ldots \stackrel{d^{\prime \prime}}{\rightarrow} \mathscr{C}_{X}^{p n} \rightarrow 0
$$

(cf. GR, p. 184). Here $\mathscr{C}_{X}^{p q}$ denotes the sheaf of germs of exterior differential forms of class $C^{\infty}$ and of bidegree $(p, q)$ on $X$. The differential $d^{\prime \prime}$ is the complex exterior differentiation operator of type $(0,1)$. 
Since the sheaves $\mathscr{E}_{X}^{p q}$ are fine, these resolutions may be used for the valculation of the cohomology groups of $\Omega_{X}^{p}$ :

$$
\begin{aligned}
& H^{q}\left(X ; \Omega_{X}^{p}\right)=H^{q}\left(\Gamma\left(\mathscr{E}_{X}^{p^{*}}\right)\right), \\
& H_{c}^{q}\left(X ; \Omega_{X}^{p}\right)=H^{q}\left(\Gamma_{c}\left(\mathcal{E}_{X}^{p^{*}}\right)\right) .
\end{aligned}
$$

We shall apply these formulae when $X$ is a polydomain in $C^{n}$, i.e. $X=U=U_{1} \times \ldots \times U_{n}$, where $U_{i}$ is a subdomain of $C$ for $i=1, \ldots, n$. We first recall the following well-known result (GR, p. 185).

Proposition 3.1. Let $U$ be a convex polydomain in $C^{n}$. Then

$$
H^{q}\left(U ; \oplus_{U}\right)=0 \text { for } q \geq 1 .
$$

In order to calculate the cohomology groups with compact supports of a convex polydomain $U$ in $C^{n}$, we consider the complex

$$
0 \rightarrow \mathscr{D}^{p^{0}} \stackrel{d^{\prime \prime}}{\rightarrow} \mathscr{D}^{p 1} \stackrel{d^{\prime \prime}}{\rightarrow} \ldots \stackrel{d^{\prime \prime}}{\rightarrow} \mathscr{D}^{p^{n}} \rightarrow 0
$$

where $\mathscr{D}^{p q}=\Gamma_{c}\left(U ; \mathscr{C}_{U}^{p q}\right)$. Derivating partially the components of a differential form we obtain the differential operators

$$
\bar{\partial}_{i}: \mathscr{D}^{p q} \rightarrow \mathscr{D}^{p q}, \quad i=1, \ldots, n .
$$

Further we define for each $i=0,1, \ldots, n$ a differential operator $d_{i}^{\prime \prime}$ : $\mathscr{D}^{p q} \rightarrow \mathscr{D}^{d, q+1}$ by

$$
d_{i}^{\prime \prime} \varphi=\sum_{j=1}^{i} d \bar{z}_{j} \wedge \bar{\partial}_{j} \varphi
$$

In particular, $d_{0}^{\prime \prime}=0$ and $d_{n}^{\prime \prime}=d^{\prime \prime}$.

Finally, we recall that the integral operators

$$
T_{i} \varphi(z)=\frac{1}{2 \pi i} \int \frac{\varphi\left(z_{1}, \ldots, \zeta, \ldots, z_{n}\right)}{\zeta-z_{i}} d \zeta \wedge d \bar{\zeta}
$$

have the property $T_{i} \bar{\partial}_{i} \varphi=\varphi=\bar{\partial}_{i} T_{i} \varphi$ for $\varphi \in \mathscr{D}^{p q}, i=1, \ldots, n \quad$ (GR, p. 25). Note, however, that the support of $T_{i} \phi$ is not compact in general.

Lemma 3.2. If $\varphi \in \mathscr{D}^{p q}$ satisfies $d_{i}^{\prime \prime} \varphi=0$ and does not involve $d \bar{z}_{1} \wedge \ldots \wedge d \bar{z}_{i}$, then $\varphi=d_{i}^{\prime \prime} \psi$ for some $\psi \in \mathscr{D}^{p, q-1}$.

Proof: If $i=0$, there are no non-zero differential forms $\varphi$ satisfying the conditions, and the assertion is trivially true. For the general case, we proceed by induction, writing

$$
\varphi=\alpha+d \bar{z}_{i} \wedge \beta
$$


where $\alpha$ and $\beta$ do not involve $d \bar{z}_{i}$. Then

$$
0=d_{i}^{\prime \prime} \varphi=d_{i-1}^{\prime \prime} \alpha+d \bar{z}_{i} \wedge\left(\bar{\partial}_{i} \alpha-d_{i-1}^{\prime \prime} \beta\right)
$$

implies $\bar{\partial}_{i} \alpha=d_{i-1}^{\prime \prime} \beta$. Applying the operator $T_{i}$, we obtain

$$
\alpha=T_{i} d_{i-1}^{\prime \prime} \beta=d_{i-1}^{\prime \prime} T_{i} \beta
$$

Let $\omega$ be a $C^{\infty}$-function depending on $z_{i}$ only, such that $\omega=1$ in a neighbourhood of the support of $\alpha$, and $\omega=0$ for $z_{i}$ outside a compact subset of $C_{i}$. Then

$$
\alpha=\omega \alpha=d_{i-1}^{\prime \prime}\left(\omega T_{i} \beta\right)
$$

where $\omega T_{i} \beta \in \mathscr{L}^{p, q-1}$.

Let us consider the difference

$$
\varphi-d_{i}^{\prime \prime}\left(\omega T_{i} \beta\right)=d \bar{z}_{i} \wedge\left(\beta-\bar{\partial}_{i}\left(\omega T_{i} \beta\right)\right) .
$$

If $\varphi$ does not involve $d \bar{z}_{1} \wedge \ldots \wedge d \bar{z}_{i}$, then $\beta$, and hence $\gamma=\beta-$ $\bar{\partial}_{i}\left(\omega T_{i} \beta\right)$, cannot involve $d \bar{z}_{1} \wedge \ldots \wedge d \bar{z}_{i-1}$. In addition, $d_{i}^{\prime \prime} \varphi=0$ implies $d_{i-1}^{\prime \prime} \gamma=0$. The induction hypothesis then shows that $\gamma=d_{i-1}^{\prime \prime} \delta$ for some $\delta \in \mathscr{D}^{p, q-2}$. Thus

$$
\varphi=d_{i}^{\prime \prime}\left(\omega T_{i} \beta-d \bar{z}_{i} \wedge \delta\right) .
$$

This completes the proof.

For the next lemma, we denote by $H_{i}, i=0, \ldots, n$, the set of functions $f \in C^{\infty}(U)$ which are holomorphic in the variables $z_{1}, \ldots, z_{i}$, or equivalently, satisfy $d_{i}^{\prime \prime} f=0$. In particular, $H_{n}$ is $\Gamma\left(U ; \mathscr{C}_{U}\right)$.

Lemma 3.3. If $\varphi \in \mathscr{D}^{n n}$ satisfies $\int f \varphi=0$ for all $f \in H_{i}$, then $\varphi=$ $d_{i}^{\prime \prime} \psi$ for some $\psi \in \mathscr{D}^{n, n-1}$.

Proof: If $i=0$, then $\int f \varphi=0$ for all $f \in C^{\infty}(U)$ implies $\varphi=0$, and the assertion holds trivially. The general case is proved by induction on $i$.

Let $\omega$ be a $C^{\infty}$-function depending on $z_{i}$ only, such that $\omega=1$ in a convex neighbourhood $C^{i-1} \times V_{i} \times C^{n-i}$ of the support of $\varphi$ and (') $=0$ for $z_{i}$ outside a compact subset of $U_{i}$.

Writing $\varphi=d \bar{z}_{i} \wedge \alpha$, we then obtain

$$
\begin{aligned}
d_{i}^{\prime \prime}\left(\omega T_{i} \alpha\right) & =d \bar{z}_{i} \wedge \bar{\partial}_{i}\left(\omega T_{i} \alpha\right) \\
& =\omega d \bar{z} \wedge \alpha+\bar{\partial}_{i} \omega d \bar{z}_{i} \wedge T_{i} \alpha=\varphi+\bar{\partial}_{i} \omega T_{i} \varphi .
\end{aligned}
$$

Hence it suffices to show that $\bar{\partial}_{i} \omega T_{i} \varphi=d_{i}^{\prime \prime} \beta$ for some $\beta \in \mathscr{D}^{n, n-1}$. 
We claim that $\int f \bar{\partial}_{i} \omega T_{i} \varphi=0$ for $f \in H_{i-1}$. For each pair of complex numbers $\zeta, \zeta^{\prime}$, the function

$$
g_{\zeta \zeta^{\prime}}(z)=\frac{1}{2 \pi i} f \bar{\partial}_{i} \omega\left(z_{1}, \ldots, \zeta^{\prime}, \ldots, z_{n}\right) \frac{1}{z_{i}-\zeta}
$$

is integrable, and

$$
\int f \bar{\partial}_{i} \omega T_{i} \varphi=\int J(\zeta, \zeta) d \zeta \wedge d \bar{\zeta}
$$

where

$$
J\left(\zeta, \zeta^{\prime}\right)=\int g_{\zeta \zeta^{\prime}} \varphi
$$

Now $g_{\xi^{\prime}} \in H_{i}$, and hence $J\left(\zeta, \zeta^{\prime}\right)=0$, if $\zeta$ is not in $U_{i}$. By analytic continuation this implies $J\left(\zeta, \zeta^{\prime}\right)=0$ for $\zeta$ outside the convex set $V_{i}$. On the other hand $g_{\zeta \zeta^{\prime}}=0$ if $\zeta^{\prime} \in V_{i}$, since $\bar{\partial}_{i} \omega=0$. Hence $J(\zeta, \zeta)=0$ for all $\zeta \in G$ concluding the proof of $\int f \bar{\partial}_{i} \omega T_{i} \varphi=0$.

The induction assumption then implies $\bar{\partial}_{i} \omega T_{i} \varphi=d_{i-1}^{\prime \prime} \beta$, and hence $d_{i}^{\prime \prime} \beta$, if the term of $\beta$ which does not involve $d \bar{z}_{i}$ is omitted. This completes the proof.

Let $C(U)$ denote the vector space of all continuous complex-valued functions defined in $U$. With the topology of uniform convergence on compact subsets of $U$, the space $C(U)$ becomes a Fréchet space. Its topological dual consists of measures with compact support on $U$ (Bourbaki [1], p. 73).

The space $\Gamma\left(U ; \widehat{C}_{U}\right)$ of holomorphic functions on $U$ is a closed subspace of $C(U)$. Hence it is a Fréchet space. Its topological dual is denoted by $\Gamma\left(U ; \mathscr{Q}_{U}\right)^{\prime}$.

Proposition 3.4. Let $U$ be a convex polydomain in $C^{n}$. Then $H_{c}^{p}\left(U ; \Omega_{U}^{n}\right)=0$ for $p \neq n$, and $H_{c}^{n}\left(U ; \Omega_{U}^{n}\right)$ is canonically isomorphic to $\Gamma\left(U ;{ }^{\Theta}\right)^{\prime}$.

Proof: Lemma 3.2 with $i=n$ implies $H_{c}^{p}\left(U ; \Omega_{U}^{n}\right)=0$ whenever $p \neq n$.

To show that $H_{c}^{n}\left(U ; \Omega_{U}^{n}\right)$ is isomorphic to the dual of $\Gamma\left(U ; \oplus_{U}\right)$ we first define a pairing

$$
\Gamma\left(U ; \mathscr{\Theta}_{U}\right) \otimes \mathscr{D}^{n n} \rightarrow C
$$

by $(f, \varphi) \rightarrow \int f \varphi$. By Lemma 3.3 this induces an injective map

$$
H_{c}^{n}\left(U ; \Omega_{U}^{n}\right) \rightarrow \Gamma\left(U ; \mathscr{\Theta}_{U}\right)^{\prime} .
$$


To prove the surjectivity, we consider a continuous linear form $u: \Gamma\left(U ; \mathscr{\Theta}_{U}\right) \rightarrow C$. By the Hahn-Banach theorem, $u$ may be extended to a continuous linear form on $C(U)$. Hence there is a compact measure $\mu$ on $U$ such that

$$
u(f)=\int f d \mu
$$

for each $f$ in $\Gamma\left(U ; \mathscr{C}_{U}\right)$. To complete the proof, we show that there is a $C^{\infty}$-measure with the same property.

Let $\delta$ denote the distance from the support of $\mu$ to the boundary of $U$. Let $\varepsilon$ be a $C^{\infty}$-function of a real variable such that

$$
\int_{0}^{\delta} r \varepsilon\left(r^{2}\right) d r=\frac{1}{2 \pi}
$$

and $\varepsilon(x)=0$ for $x \geq \delta^{2} / 2 n$, and define a $C^{\infty}$-function $\omega$ by

$$
\omega(z)=\varepsilon\left(\left|z_{1}\right|^{2}\right) \ldots \varepsilon\left(\left|z_{n}\right|^{2}\right) .
$$

Let $f$ be a holomorphic function in $U$. Then, by the mean value theorem

$$
f(z)=\int \omega(z-\zeta) f(\zeta) d \tau
$$

where $\tau$ is the Lebesgue measure, at each point $z$ of $\operatorname{supp} \mu$. Hence

$$
\varphi(z)=\int \omega_{z} d \mu
$$

where $\omega_{z}(\zeta)=\omega(\zeta-z)$, is a $C^{\infty}$-function such that

$$
\int f d \mu=\int f \varphi d \tau
$$

and $\operatorname{supp} \varphi$ is a compact subset of $U$. This completes the proof.

\section{Duality for coherent sheaves}

In this section we continue to assume that $X$ is a complex analytic manifold of dimension $n$. Our aim is to give a generalization of the duality of Proposition 3.4 for coherent sheaves. This is done in two cases: in Theorem 4.5 for compact manifolds and in Theorem 4.6 for Stein manifolds.

In the preceding section we have seen that the exterior product of differential forms induces a map

$$
H^{p}\left(X ; \Theta_{X}\right) \otimes H_{c}^{q}\left(X ; \Omega_{X}^{n}\right) \rightarrow H_{c}^{p+q}\left(X ; \Omega_{X}^{n}\right) .
$$


It is not difficult to show that this agrees, up to sign, with the composition product

$$
\operatorname{Ext}_{O_{X}}^{p}\left(\mathscr{\Theta}_{X}, \mathscr{\Theta}_{X}\right) \otimes \operatorname{Ext}_{c, O_{X}}^{q}\left(\mathscr{\Theta}_{X}, \Omega_{X}^{n}\right) \rightarrow \operatorname{Ext}_{c, O_{X}}^{p+q}\left(\mathscr{\Theta}_{X}, \Omega_{X}^{n}\right)
$$

In case $p=0$ each product defines the natural $\Gamma\left(X ; C_{X}\right)$-module structure of $H_{q}^{c}\left(X ; \Omega_{X}^{n}\right)$. This is the only case which will be needed in the following.

The sections of $\mathscr{C O C}_{X}^{n n}$ may be considered as measures on $X$. Those with compact support are of finite total mass. Hence the integral defines a linear map $\Gamma_{c}\left(X ; \mathscr{C}_{X}^{n n}\right) \rightarrow C$. Since $\int d^{\prime \prime} \varphi=\int d \varphi=0$ for $\varphi \in \Gamma_{c}\left(X ; \mathscr{C}_{X}^{n, n-1}\right)$ by Stokes' theorem, this map may be factored through $H^{n}\left(\Gamma_{c}\left(X ; \mathscr{C}_{X}^{n}\right)\right)$. The induced homomorphism $H_{c}^{n}\left(X ; \Omega_{X}^{n}\right) \rightarrow C$ will be referred to as the trace map.

Let 7 be an $\mathscr{C}_{X}$-Module. By composing the trace map and the composition product, we obtain a pairing

$$
H^{p}(X ; \mathcal{F}) \otimes \operatorname{Ext}_{c, O_{X}}^{n-p}\left(\mathcal{F}, \Omega_{X}^{n}\right) \rightarrow C .
$$

In case $X$ is a convex polydomain in $C^{n}$ and $\mathcal{F}=\mathscr{C}_{X}$, this pairing defines an isomorphism of $\operatorname{Ext}_{c, O_{X}}^{n-p}\left(\mathscr{C}_{X}, \Omega_{X}^{n}\right)$ onto the topological dual of $H^{p}\left(X ; \circlearrowleft_{X}\right)$ by Proposition 3.4. In order to prove this duality in other cases, we extend the pairing to spectral sequences. For this purpose, $C$ is considered as a degenerate spectral sequence whose only non-vanishing terms are $E_{r}^{0 n}=C, \quad r \geq 1$.

Let $\mathfrak{U}$ be an open cover of $X$. By Corollary 1.4, there is a spectral sequence converging to $H_{c}^{*}\left(X ; \Omega_{X}^{n}\right)$, such that

$$
E_{1}^{p q}=C_{-p}\left(\mathfrak{U} ; h_{c}^{q}\left(\Omega_{X}^{n}\right)\right)
$$

where $h_{c}^{q}\left(\Omega_{X}^{n}\right)$ denotes the precosheaf $U \rightarrow H_{c}^{q}\left(U ; \Omega_{X}^{n}\right)$. To the trivial open cover, which consists of only one set $X$, there corresponds the degenerate spectral sequence $E_{r}^{0 q}=H_{c}^{q}\left(X ; \Omega_{X}^{n}\right), \quad r \geq 1$. The augmentation map

$$
C_{0}\left(\mathfrak{U} ; h_{c}^{q}\left(\Omega_{X}^{n}\right)\right) \rightarrow H_{c}^{q}\left(X ; \Omega_{X}^{n}\right)
$$

may be extended to a natural homomorphism of these spectral sequences since $\mathfrak{U}$ is a refinement of the trivial open cover. The trace map $H_{c}^{n}\left(X ; \Omega_{X}^{n}\right) \rightarrow C$ may also be regarded as a homomorphism of degenerate spectral sequences. Composing these homomorphisms, we obtain

Proposition 4.1. Let $\mathfrak{U}$ be an open cover of $X$. Then the trace map $H_{c}^{n}\left(X ; \Omega_{X}^{n}\right) \rightarrow C$ may be extended to a homomorphism of spectral sequences such that the homomorphism of $E_{1}$-terms

$$
C_{0}\left(\mathfrak{U} ; h_{c}^{n}\left(\Omega_{X}^{n}\right)\right) \rightarrow C
$$

is the sum of the trace maps $H_{c}^{n}\left(U ; \Omega_{X}^{n}\right) \rightarrow C$ where $U \in \mathfrak{U}$. 
Let $\mathcal{F}$ be a coherent analytic sheaf on $X$. We recall that there is a natural way of introducing a topology into the vector space of sections $\Gamma(U ; \mathcal{F})$ over any open subset $U$ of $X$, so that this becomes a Fréchet space $(G R, p p .237,239)$. If $\mathcal{F}=\mathscr{C}_{X}$, then the space of sections has the topology of uniform convergence on compact subsets. The maps $I(U ; 7) \rightarrow$ $\Gamma\left(U ; C_{\mathcal{G}}\right)$ induced by a homomorphism of coherent sheaves $\mathcal{I} \rightarrow$ C are continuous, as well as the restriction maps $\Gamma(U ; \mathcal{F}) \rightarrow \Gamma(V ; \mathcal{F})$ for open sets $V \subset U \subset X$. This implies the continuity of the differentials of the complex $C^{\cdot}(\mathfrak{U} ; \mathcal{F})$ for any open cover $\mathfrak{U}$ of $X$.

In order to deal with the duality of topological vector spaces, we reproduce two lemmas of Serre [5].

Lemma 4.2. Let $E, F$, and $G$ be Fréchet spaces. Let $u: E \rightarrow F$ and $v: F \rightarrow G$ be continuous linear maps such that $v \circ u=0$. Let $u^{\prime}: F^{\prime} \rightarrow E^{\prime}$ and $v^{\prime}: G^{\prime} \rightarrow F^{\prime}$ denote the adjoint maps of $u$ and $v$.

Then, if $\operatorname{im} u$ and $\operatorname{im} v$ are closed, $H=\operatorname{ker} v / \operatorname{im} u$ is a Fréchet space, and its topological dual $H^{\prime}$ is isomorphic to $\operatorname{ker} u^{\prime} / \operatorname{im} v^{\prime}$.

Proof: Since the quotient of a Fréchet space by a closed subspace is a Fréchet space, the first assertion follows immediately.

Let $f: F \rightarrow C$ be an element of ker $u^{\prime}$. Then $f(\operatorname{im} u)=0$, and $f$ defines a continuous linear form $h$ on $H$. If $f \in \operatorname{im} v^{\prime}$, then $f(\operatorname{ker} v)=0$, and hence $h=0$. Inversely, if $h=0$, then $f$ is induced by a linear form $g_{1}$ on im $v$. By the open mapping theorem, $g_{1}$ is continuous. Hence it may be extended to a continuous linear form $g$ on $G$ by the Hahn-Banach theorem. Then $f=v^{\prime}(g) \in \operatorname{im} v^{\prime}$. Thus $\operatorname{ker} u^{\prime} / \operatorname{im} v^{\prime}$ may be identified with a subspace of $H^{\prime}$.

On the other hand, each element of $H^{\prime}$ defines a continuous linear form on $\operatorname{ker} v$, and this may be extended to $F$ by the Hahn-Banach theorem. Hence each continuous linear form on $H$ is induced by an element of $\operatorname{ker} u^{\prime}$. This completes the proof.

Lemma 4.3. Let $E$ and $F$ be Fréchet spaces and $u: E \rightarrow F$ a continuous linear map. If the dimension of $F / \operatorname{im} u$ is finite, then im $u$ is closed in $F$.

Proof: Let $G$ denote $F / \operatorname{im} u$ with the natural Hausdorff topology, and let $v$ be a right inverse to the projection $F \rightarrow G$. Then $v$ is continuous, and $E \times G$ is a Fréchet space. The map $w=(u, v)$ from $E \times G$ to $F$ is surjective, and hence open by the open mapping theorem. Since $w^{-1}(\operatorname{im} u)=E$ is closed, im $u$ is closed in $F$.

We now prove a preliminary form of the duality theorem. 
Proposition 4.4. Let $X$ be a holomorphically convex open subset of $C^{n}$. Let $\mathcal{F}$ be a coherent analytic sheaf on $X$ such that there is a finite free resolution

$$
0 \rightarrow \mathscr{C O}_{X}^{i_{k}} \rightarrow \mathscr{C O}_{X}^{i_{k-1}} \rightarrow \ldots \rightarrow \mathscr{C}_{X}^{i_{0}} \rightarrow \mathcal{F} \rightarrow 0
$$

Then $\operatorname{Ext}_{c, O_{X}}^{p}\left({ }^{7}, \Omega_{X}^{n}\right)=0$ for $p \neq n$ and the pairing

$$
H^{0}(X ; \mathcal{F}) \otimes \operatorname{Ext}_{c, O_{X}}^{n}\left(\mathcal{F}, \Omega_{X}^{n}\right) \rightarrow C
$$

defines an isomorphism of $\operatorname{Ext}_{c, O_{X}}^{n}\left(\mathcal{F}, \Omega_{X}^{n}\right)$ onto the topological dual of $\Gamma(X ; \mathcal{\exists})$.

Proof: We assume first that $\mathcal{F}=\Theta_{X}$. Let $\mathfrak{U}$ be a countable family of convex polydomains covering $X$. By Proposition 1.1, there is a spectral sequence $E$ converging to $H^{\cdot}\left(X ; \Theta_{X}\right)$, such that

$$
E_{1}^{p q}=C^{p}\left(\mathfrak{U} ; h^{q}\left({ }^{(}{ }_{X}\right)\right),
$$

where $h^{q}\left(\mathscr{C}_{X}\right)$ denotes the presheaf $U \rightarrow H^{q}\left(U ; \mathscr{C}_{X}\right)$. Since $H^{q}\left(U ; \mathscr{C}_{X}\right)=0$ for $q>0$ whenever $U$ is a convex polydomain, we have $E_{1}^{p q}=0$ for $q>0$. On the other hand, each $E_{1}^{p^{0}}$ is a countable product of Fréchet spaces, hence a Fréchet space.

By Corollary 1.4, there is a spectral sequence ${ }_{c} E$ converging to Ext $_{c, O_{X}}^{\cdot}\left(\circlearrowleft_{X}, \Omega_{X}^{n}\right)=H_{c}^{\cdot}\left(X ; \Omega_{X}^{n}\right)$ such that

$$
{ }_{c} E_{1}^{p q}=C_{-p}\left(\mathfrak{U} ; h_{c}^{q}\left(\Omega_{X}^{n}\right)\right) .
$$

From Proposition 3.4 we infer that ${ }_{c} E_{1}^{p q}=0$ for $q \neq n$, and hence ${ }_{c} E_{2}^{p n} \cong H_{c}^{p+n}\left(X ; \Omega_{X}^{n}\right)$.

Composing the pairing $E \otimes{ }_{c} E \rightarrow{ }_{c} E$ of Theorem 2.2 and the homomorphism ${ }_{c} E \rightarrow C$ of Proposition 4.1, we obtain a pairing of spectral sequences

$$
E \otimes{ }_{c} E \rightarrow C
$$

By Proposition 3.4 this pairing defines an isomorphism of ${ }_{c} E_{1}^{-p, n}$ onto the topological dual of $E_{1}^{p^{0}}$. Since $H^{p}\left(E_{1}^{\cdot 0}\right)=E_{2}^{p^{0}} \cong H^{p}\left(X ;{ }^{(}{ }_{X}\right)=0$ for $p>0$, Lemma 4.2 implies that $H^{p}\left({ }_{c} E_{1}^{\cdot n}\right)={ }_{c} E_{2}^{p n}=0$ for $p<0$ and ${ }_{c} E_{2}^{0 n}=H_{c}^{n}\left(X ; \Omega_{X}^{n}\right)$ is isomorphic to the dual of $H^{0}\left(E_{1}^{\cdot 0}\right) \cong H^{0}\left(X ; \mathscr{C}_{X}\right)$. This proves the proposition in case $\mathcal{F}=\mathscr{C}_{X}$.

To complete the proof, we proceed by induction on the length $k$ of the resolution of 7 . The case $k=0$ is settled by the additivity of the functors $H^{\circ}$ and $\mathrm{Ext}_{c}^{\circ}$.

Let us assume that the assertion is true for the kernel ' $?$ of the epimorphism $\mathbb{C}_{X}^{i_{0}} \rightarrow \mathcal{7}$. The sequence of sections

$$
0 \rightarrow \Gamma\left(X ; C_{Y}\right) \rightarrow \Gamma\left(X ;\left(C_{X}^{i_{0}}\right) \rightarrow \Gamma(X ; \overline{\mathcal{J}}) \rightarrow 0\right.
$$


is exact, since $X$ is holomorphically convex. By Lemma 4.2 its dual sequence

$$
0 \rightarrow \Gamma(X ; \mathcal{J})^{\prime} \rightarrow \Gamma\left(X ; \mathscr{C}_{X}^{i_{0}}\right)^{\prime} \rightarrow \Gamma\left(X ; C_{1}\right)^{\prime} \rightarrow 0
$$

is also exact. By comparing this with the exact sequence of Ext ${ }_{c}$, we see that the assertion holds for $\mathcal{F}$.

Theorem 4.5. Let $X$ be a compact complex analytic manifold of dimension $n$. Let $\exists$ be a coherent analytic sheaf on $X$. Then $\operatorname{Ext}_{c, O X}^{n-p}\left(\mathcal{F}, \Omega_{X}^{n}\right)$ is canonically isomorphic to the dual of $H^{p}(X ; \mathcal{J})$.

Note that the space $H^{p}(X ; \mathcal{F})$ is of finite dimension.

Proof: Since $\mathcal{F}$ is coherent, for each point $x$ of $X$ there is an open neighbourhood $U$ such that $\mathcal{F} \mid U$ has a finite free resolution (GR, p. 138). Let $\mathfrak{U}$ be a finite family of holomorphically convex coordinate neighbourhoods with this property covering $X$.

Let us consider the spectral sequences $E$ and ${ }_{c} E$ of $H^{\cdot}(X ; \overline{7})$ and $\operatorname{Ext}_{c, O_{X}}\left(\mathcal{F}, \Omega_{X}^{n}\right)$ with respect to $\mathfrak{H}$. Composing the pairing of Theorem 2.2 with the homomorphism of Proposition 4.1, we obtain a pairing of spectral sequences $E \otimes_{c} E \rightarrow C$. Since finite intersections of holomorphically convex open sets are holomorphically convex, we infer by Proposition 4.4 that this pairing defines an isomorphism of

$$
{ }_{c} E_{1}^{-p, n}=C_{p}\left(\mathfrak{l} ; \operatorname{ext}_{c, O_{X}}^{n}\left(\mathcal{F}, \Omega_{X}^{n}\right)\right)
$$

onto the topological dual of $E_{1}^{p^{0}}=C^{p}(\mathfrak{U} ; \mathcal{F})$, and that the other terms vanish. Then Lemma 4.2 implies that

$$
\operatorname{Ext}_{c, \mathcal{O}_{X}}^{n-p}\left(\mathcal{F}, \Omega_{X}^{n}\right)={ }_{c} E_{2}^{-p, n}=H_{p}\left(\mathfrak{H} ; \operatorname{ext}_{c, O_{X}}^{n}\left(\mathcal{F}, \Omega_{X}^{n}\right)\right)
$$

is isomorphic to the dual of $H^{p}(X ; \overline{\mathcal{I}})=E_{2}^{p 0}=H^{p}\left(\mathfrak{U} ;{ }^{\mathcal{F}}\right)$ provided the differentials of the complex $C^{*}(\mathfrak{U} ; \mathcal{F})$ have closed range. But this follows from Lemma 4.3 , since $H^{p}(X ; \mathcal{J})$ is finite dimensional by the compactness of $X$ (GR, p. 245).

Theorem 4.6. Let $X$ be a Stein manifold of complex dimension $n$. Let $\mathcal{I}$ be a coherent analytic sheaf on $X$. Then $\operatorname{Ext}_{c, O_{X}}^{p}\left(\mathcal{F}, \Omega_{X}^{n}\right)=0$ for $p \neq n$ and $\operatorname{Ext}_{c, O_{X}}^{n}\left(\mathcal{F}, \Omega_{X}^{n}\right)$ is isomorphic to the topological dual of $H^{0}(X ; \mathcal{F})$.

Proof: The topology of a Stein manifold has a countable base by definition. Hence $X$ may be covered with a countable family $\mathfrak{H}$ of open holomorphically convex coordinate neighbourhoods $U$ with the property that ${ }^{\mathcal{T}} U$ has a finite free resolution. The proof is then completed as in the first part of Proposition 4.4, noting that $H^{p}(X ; \mathcal{F})=0$ for $p>0$ by Cartan's Theorem B (GR, p. 243).

Department of mathematics,

University of Helsinki. 


\section{References}

[1] Bourbaki, N.: Intégration, chap. 1-4, Hermann, Paris, 1952.

[2] Cartier, P.: Les groupes $\operatorname{Ext}^{5}(\mathrm{~A}, \mathrm{~B})$, Séminaire Grothendieck, t. 1, 1957, $\mathrm{n}^{\circ} 3$, Secrétariat mathématique, Paris, 1958.

G Godement, R.: Topologie algébrique et théorie des faisceaux, Hermann, Paris, 1958. GR Gunning, R. C.-Rossi, H.: Analytic functions of several complex variables, Prentice-Hall, Englewood Cliffs, N.J., 1965.

[3] Hartshorse, R.: Residues and duality, Springer, Berlin-Heidelberg-New York, 1966.

[4] Malgrange, B.: Systemes différentiels a coefficients constants, Séminaire Bourbaki, t. $15,1962 / 63, \mathrm{n}^{\circ} 246$, Secrétariat mathématique, Paris, 1964.

[5] Serre, J.-P.: Un théoreme de dualité, Comm. Math. Helv. 29, 1955, 9-26.

[6] SpAxier, E.H.: Algebraic topology, McGraw-Hill, New York, 1966. 